\title{
Cadena productiva y capital social: el caso de la piscicultura del Cauca, Colombia*
}

Productive chain and social capital: the case of fish farmers of Cauca, Colombia

Cadeia produtiva e capital social: o caso dos piscicultores de Cauca, Colômbia

La chaîne de production et capital social: le cas de la pisciculture du Cauca, Colombie

\author{
Elizabeth Ruano** \\ (elizabeth@unb.br) \\ Valcilon Silva*** \\ (valcilon@unb.br) \\ Wilfred Rivera** \\ (wrivera@uniautonoma.edu.co)
}

Recebido em 26/03/2015; revisado e aprovado em 24/07/2015; aceito em 31/07/2015

DOI: http:/ / dx.doi.org/10.1590/151870122015202

\begin{abstract}
Resúmen: Este texto analiza la adopción del enfoque de cadena productiva en la piscicultura del Cauca, Colombia. Se trata de un estudio cualitativo apoyado en la investigación acción y en entrevistas abiertas. El análisis retoma los conceptos de cadena productiva y capital social. El análisis confirmó el fortalecimiento de redes relacionales como requisito fundamental para generar capacidades empresariales y tecnológicas en la piscicultura caucana. Palabras clave: Capital social. Cadena productiva. Piscicultura-Cauca-Colombia.

Resumo: Neste trabalho se analisa a tentativa de adoção do enfoque de cadeia produtiva na piscicultura de Cauca, Colômbia. Trata-se de um estudo qualitativo voltado para a pesquisa-ação e entrevistas abertas. Na análise retomam-se os conceitos de cadeia produtiva e capital social. Os resultados evidenciam que o fortalecimento das redes relacionais entre os agentes constituíram uma etapa fundamental para gerar capacidades empresariais e tecnológicas na atividade piscícola dessa região.

Palavras-chave: Capital social. Cadeia produtiva. Piscicultura-Cauca-Colômbia.

Abstract: This paper analyzes the adoption of the approach used on the aquaculture production chain in Cauca, Colombia. It is about a qualitative study supported in participatory action research and open interviews. The analysis retakes the concepts of supply chain and social capital. The results confirmed that the strengthening of networks of relationships as a fundamental requisite to generate entrepreneurial and technological capabilities on Cauca's aquaculture.

Key words: Social capital. Productive chain. Aquaculture-Cauca-Colombia.

Résumé: Cette article analyse l'adoption de chaîne de production dans la pisciculture du Cauca en Colombie. Il s'agit d'une étude qualitative basée sur la recherche, l'action et sur des entretiens. L'analyse reprend les concepts de chaîne productive et de capital social. Cette analyse a confirmé que les réseaux de relations sont une condition indispensable pour générer des compétences entrepreneuriales et technologiques dans le secteur piscicole du Cauca. Mots clés: Capital social. Chaîne de production. Pisciculture-Cauca-Colombie.
\end{abstract}

\section{Introducción}

Este trabajo analiza el proceso mediante el cual los agentes vinculados a la piscicultura del Cauca, región localizada al sur de Colombia, intentaron adoptar del enfoque de cadena productiva. La promoción de ese enfoque a la piscicultura, en particular, y en general a otros sectores productivos de pequeña escala, constituyó una estrategia gubernamental colombiana ejecutada sistemáticamente a partir de la década de 1990. La promulgación de la Ley $n^{\circ} 811$ de 2003, conocida como Ley de Cadenas Productivas, fue el marco jurídico que legitimó esa iniciativa gubernamental.

La promoción del enfoque de cadena productiva por el gobierno colombiano inició a partir de 1996, cuando los sectores textil, siderúrgico, metalmecánico y de la industria gráfica, fueron pioneros en adoptar dicha perspectiva. Posteriormente, los sectores avícola, porcino, lechero y acuícola lo promovieron

\footnotetext{
* Este manuscrito se elaboró a partir de los datos empíricos obtenidos en marco del proyecto titulado "Fortalecimiento de la piscicultura del Cauca", financiado por el Programa Colombia de la Universidad Georgetown y la Agencia para el Desarrollo Internacional de los Estados Unidos (USAID) y ejecutado por el Centro Regional de Productividad e Innovación del Cauca (Crepic).

** Corporación Universitaria Autónoma del Cauca, Cauca, Colombia.

*** Universidade de Brasília, Brasília, Distrito Federal, Brasil.
} 
como estrategia de fortalecimiento. Entre los años 1998 a 2002, el fomento estatal de las cadenas productivas regionales, constituyó el eje central de la política pública para la productividad y competitividad (COLOMBIA, 2003).

A partir del 2003, el Centro Regional de Productividad e Innovación del Cauca (Crepic) con financiamiento del Programa Colombia de la Universidad Georgetown, en concordancia con las disposiciones de la Ley $\mathrm{n}^{\circ} 811$ de 2003, inició un proyecto de fortalecimiento de la piscicultura del Cauca ${ }^{1}$. Esa actividad productiva había sido identificada como prioritaria en diferentes ejercicios de planeación territorial adelantados desde la década de $1990^{2}$. El perfil étnico de los piscicultores - mayoritariamente afrodescendientes e indígenas -, la significativa expansión territorial de esa actividad a 30 de los $42 \mathrm{mu}$ nicipios que componen el Cauca, el potencial en recursos hídricos y condiciones climáticas favorables son criterios que orientaron esa priorización. Dicho proyecto fue orientado a mejorar la competitividad piscícola del Cauca tomando el enfoque de cadena productiva como referente de actuación. También incentivó la utilización de instrumentos de la investigación acción (FALS BORDA, 1992; ORTIZ; BORJAS, 2008) direccionados a la sistematización de los aprendizajes del proyecto.

\footnotetext{
${ }^{1}$ En el año 2005, la región tenía 1.268.937 habitantes, $60 \%$ de ellos en el ámbito rural. En ese año alcanzó $1,7 \%$ del PIB nacional, en el PIB caucano se destacan el sector industrial y el gubernamental contrastando con el rezado del sector agropecuario. Ocupa el segundo lugar a nivel nacional por la desigualdad en la distribución de la tierra. A pesar de la diversidad de suelos, solamente el $25,1 \%$ pueden destinarse a la producción agrícola y forestal, 36, 4\% deben dedicarse a la conservación forestal y agroforestal. Más de la mitad de los suelos no son aptos para la agricultura, $32 \%$ son de baja fertilidad y $25 \%$ de muy baja fertilidad. Adicionalmente persisten las restricciones de infraestructura como carreteras, energía eléctrica, agua potable, entre otros. La mayoría de sus municipios registran un porcentaje de necesidades básicas insatisfechas entre 50 y $80 \%$ (GAMARRA, 2007).

2 Plan de Ciencia y Tecnología del Pacífico Colombiano (1996); Visión Cauca 2020 (1999), Comité Asesor de Comercio Exterior - CARCE (2001), Agenda Caucana de Ciencia y Tecnología - CAUCACYT (2003), Agenda Interna para la productividad y la competitividad del Cauca (2004), Plan Regional de Competitividad del Cauca (2007), Visión Cauca 2032 (2010), Pacto por la Innovación (2011), Construcción Plan Departamental de Desarrollo 2012-2015 (2012), Contrato Plan (2013) y CONPES Cauca (2013).
}

Este artículo retoma datos empíricos a la luz de los conceptos de cadena productiva y capital social. Las siguientes preguntas orientaron el análisis aquí presentado: ¿Cómo se dinamizó la adopción del enfoque de cadena productiva entre los agentes de la piscicultura del Cauca? ¿Cuáles fueron los avances más relevantes en el proceso de transición del enfoque de subsistencia para el enfoque de cadena productiva en la piscicultura del Cauca? Este trabajo incluye tres subtítulos además de esta introducción. En la primera parte se describe la metodología y los conceptos de referencia. Enseguida se analizan las tensiones y avances del proceso estudiado. Para concluir se incluyó un ítem dedicado a las consideraciones finales.

\section{El enfoque de cadena productiva como objeto de estudio}

Analizar el proceso emprendido por los agentes de la piscicultura del Cauca, Colombia, se justifica porque ese caso permite cuestionar las especificidades que surgen durante una pretendida transición del enfoque de subsistencia hacia la perspectiva de cadena productiva. Mediante ese proceso los agentes locales buscaron encuadrarse en los lineamentos de una política pública de carácter nacional. Después de la expedición de la Ley $n^{\circ} 811$ de 2003, denominada 'Ley de cadenas productivas', varios sectores productivos colombianos, en su mayoría minifundistas, iniciaron procesos de reorganización bajo dicha perspectiva (COLOMBIA, 2003).

Abordar el proceso de organización de la cadena piscícola del Cauca como objeto de estudio permite indagar sobre el potencial del concepto de cadena productiva en la definición de políticas públicas para sectores productivos con carácter de subsistencia. En el debate académico, Morvan (1985) defendió dicho potencial. Piñones et al. (2006), defienden que la adopción del enfoque de cadena productiva vía políticas públicas contribuye mejorando la distribución del ingreso en áreas rurales, aumentando los niveles de competitividad de los diferentes agentes y eslabones de la cadena. Para esos autores, el concepto de cadena productiva destaca las relaciones de agregación de valor entre los agentes de producción, comercialización y consumo de un producto agropecuario. 
Según Tomta y Chiatchoua (2009, p. 149), el objetivo principal del enfoque de cadena productiva consiste en "localizar las empresas, las instituciones, las operaciones, capacidades de negociación, las tecnologías, las relaciones de producción y las relaciones de poder en la determinación de los precios". Para Morvan (1985), las relaciones entre los agentes del encadenamiento están determinadas por fuerzas jerárquicas, enfatiza la jerarquía y poder del mercado y de la acción gubernamental directa o indirectamente al encadenamiento productivo.

En el debate académico se buscó que el enfoque de cadena productiva superara la visión estática y secuencial incorporando una perspectiva no lineal, dinámica, colaborativa y sistemática. Sin embargo, la racionalidad económica continúa como determinante de las relaciones entre los agentes. En ese sentido, el establecimiento de precios competitivos, la agregación de valor mediante la apropiación de conocimiento, la inserción en el mercado y el aumento de las ganancias delimitarían las relaciones entre los agentes (TOMTA; CHIATCHOUA, 2009).

De manera amplia, el concepto cadena productiva refiere un sistema relativamente autónomo y capaz de asegurar su propia transformación mediante una secuencia de operaciones que conducen a la producción de bienes o servicios. Esa producción es ampliamente influenciada por una frontera de posibilidades que es definida, principalmente, por la tecnología y por las estrategias de los agentes que buscan maximizar sus ganancias (MORVAN, 1985).

En la ley colombiana la cadena productiva fue definida como un espacio de encuentro que surge de una libre decisión de sus integrantes de aliarse para mejorar su competitividad, con el fin de obtener un mejor desempeño técnico, económico individual y colectivo. Esa decisión requería ser formalizada mediante la firma de un acuerdo de competitividad (COLOMBIA, 2003). El enfoque de cadena productiva fue promocionado como una estrategia de diferenciación para acceder a recursos públicos para enfrentar problemas comunes a varios sectores productivos.

En el Cauca el enfoque de cadena productiva recibió significativa acogida. Tal receptividad se explica, en primer lugar, porque la referida ley estableció que el financiamiento de proyectos productivos, con recursos del gobierno nacional, estaría condicionado a la existencia de los acuerdos de competitividad. De otro lado, la importante demanda por recursos públicos del orden nacional se apoya en diagnósticos oficiales (COLOMBIA, 2004b) que revelaron una crítica situación financiera del Departamento del Cauca y entrabes financieros, tecnológicos y mercadológicos en los sectores productivos representativos de la región.

En los ejercicios gubernamentales de planeación territorial, realizados entre 1996 a 2013, la piscicultura fue priorizada como apuesta estratégica del Cauca. Tal priorización, liderada por el gobierno local con la participación de diversos agentes locales, posicionó a la piscicultura como un sector productivo con potencial para estimular el desarrollo de la región (COLOMBIA, 2004c). Sin embargo, los piscicultores del Cauca son, principalmente, afrodescendientes e indígenas que poseen unidades productivas de subsistencia que no superan cinco hectáreas de extensión.

En el Cauca, según declaraciones de los piscicultores, la piscicultura se inició en los años 80 , mediante una iniciativa estatal para regiones vulnerables al conflicto armado y a la expansión de cultivos de uso ilícito. En ese sentido, la piscicultura del Cauca inició gracias al asistencialismo estatal que estimuló esa cultura productiva con carácter de subsistencia. En el resto del país la explotación comercial de la piscicultura se estableció una década antes, al final de la década de los años sesenta (COLOMBIA, 2004a; ESPINAL, 2005).

De otro lado, los estudios del capital social en contextos rurales han destacado el potencial explicativo de las nociones como reciprocidad, cooperación y confianza entre los agentes. La incorporación de esas dimensiones en el análisis aquí presentado permite relativizar la centralidad de la racionalidad económica presente en el concepto de cadena productiva (FORONDA; GALINDO, 2012). El pretendido proceso de transición de la piscicultura de subsistencia hacia el enfoque de cadena productiva puede ser mejor comprendido a la luz de la noción de capital social en contextos rurales.

La importancia del capital social fue discutida por Foronda y Galindo (2012, p. 
46), a partir del contexto español. Se trata de un concepto académico que enriquece la comprensión de las dinámicas socioeconómicas de los territorios rurales. El capital social, en contextos rurales, puede definirse como una "habilidad para acceder a una serie de recursos que es facilitada por el hecho de estar incluido en una "red estable" de relaciones personales y sociales". Esas redes presentan valores de pertenencia y adscripción que van a marcar la cohesión entre agentes, sean estos individuos o instituciones.

No es factible reproducir aquí la amplia discusión en torno del concepto de capital social. Sin embargo, conviene citar que en el enfoque estructural (BOURDIEU, 1984), destaca la totalidad de los recursos, actuales o potenciales, de los agentes que integran redes relativamente institucionalizadas de conocimiento y reconocimiento mutuo. La movilidad de esos recursos ocurre a través de acciones intencionadas de los agentes. La confianza y compromiso con las normas por parte de los agentes y grupos se dinamizan a partir de objetivos y acciones conjuntos. De ese modo, el capital social se potenciaría a partir del dinamismo de las relaciones entre los agentes.

De otro lado, en el enfoque culturalista (PUTNAM, 1993), el capital social se aborda como un fenómeno subjetivo compuesto por valores éticos como la reciprocidad, cooperación y compromisos y también por valores sociales como la confianza en el poder individual para transformar su entorno, el sentido de pertenencia, arraigo e identidad. En ese sentido, el capital social sería un atributo individual que se expresa en las redes de reciprocidad a las cuales tienen acceso los agentes.

Según Putnam (1993), el capital social contribuye a sumar esfuerzos para fines comunes, a pesar de las diferencias de origen socioeconómico y cultural de los agentes. En ese orden, la confianza se revela como un fenómeno cultural colectivo resultante de la acumulación y actualización de experiencias colectivas o individuales propiciadas en ambientes de socialización (PUTNAM, 2002). Por eso, el capital social seria portátil, es decir, los individuos lo llevan consigo a donde quieran. Siendo así, el capital social es plausible de ser inculcado.
En este trabajo se utiliza el concepto de capital social en contextos rurales para destacar la centralidad de los valores de pertenecía y adscripción en la cohesión entre los agentes que se requiere en el enfoque de cadena productiva. Aquí se presenta el análisis detallado de un único caso, centrado en la particularización de la piscicultura del Cauca. Es decir, constituye una investigación típicamente cualitativa (BOLSEGUÍ; FUGUET, 2006). El análisis de ese caso, permite contrastar el potencial y desafíos del enfoque de cadena productiva en las políticas públicas para sectores productivos con carácter de subsistencia en Colombia.

El abordaje metodológico incluyó la realización de 21 entrevistas abiertas a interlocutores clave que ostentaban una década de experiencia, o más, en la producción piscícola caucana. A los entrevistados fue solicitado que identificaran las dificultades, las trasformaciones y resultados del proyecto que dinamizó el proceso de transición de la subsistencia para el enfoque de cadena productiva. Todos los entrevistados corresponden al sexo masculino. 14 de ellos, en esa época, poseían escolaridad básica y su edad oscilaba entre 40 y 60 años. Siete entrevistados se desempeñaban como representantes de las Secretarías departamental y municipal de Agricultura, tenían entre 30 y 55 años de edad, amplia experiencia en piscicultura y poseían título universitario.

También se retoman los datos sistematizados en marco del proyecto de fortalecimiento de la piscicultura del Cauca iniciado a partir del año 2003. El análisis retoma la primera etapa del proyecto la cual se finalizó en julio del 2006. En ella se promovió la realización de reuniones como mecanismo de aproximación entre los actores convocados. Según Comerford (2002), las reuniones crean espacios de sociabilidad que ayudan en la consolidación de redes de relaciones y accionan múltiples concepciones sobre los temas en pauta. En total fueron realizadas 20 reuniones que permitieron la observación, el dialogo informal, la reflexión sobre la práctica y la planificación de acciones frente a los problemas identificados. Cada una de esas reuniones alcanzó una duración de seis horas continuas de trabajo conjunto.

De esas reuniones participaron un total de 130 agentes: 80 piscicultores de pequeña 
escala, 10 proveedores de insumos, cinco comercializadores, 20 entidades públicas y 15 entidades privadas. Durante las reuniones, el saber empírico de los piscicultores buscó conjugarse con el conocimiento técnico de los agentes externos, de orden gubernamental y privado. Para facilitar la interacción entre los agentes y validar la información se formaron cuatro grupos temáticos de discusión: asociatividad, financiamiento, comercialización y tecnología productiva. Los datos primarios obtenidos en las reuniones fueron complementados con los datos oficiales disponibles en los censos agropecuarios. De ese modo, se avanzó en la elaboración de un diagnóstico de la piscicultura del Cauca.

Al tornar el enfoque de cadena productiva como objeto de estudio se observa que esa dinámica presenta de un lado, estrategias utilizadas en la promoción gubernamental de su política pública y de otro lado, estrategias de los agentes de la piscicultura caucana que buscaron respuestas para los problemas de su actividad productiva. Si bien esos intereses no son totalmente opuestos, para los agentes de la piscicultura caucana la adopción de ese enfoque implicó en un proceso de transición que requería recursos no disponibles. Enseguida se describen algunos momentos del proceso con énfasis en las tensiones observadas.

\section{De la subsistencia hacia el enfoque de cadena productiva: tensiones de la transi- ción pretendida}

Durante los primeros meses, segundo semestre del 2003, se hizo evidente cierta incompatibilidad respecto a la temporalidad del proyecto. Para los investigadores, el tiempo aparecía asociado, principalmente, al cronograma a ser cumplido. Para los piscicultores, ese cálculo remitía a un balance entre las expectativas de resultados y concretos frente al tiempo invertido en las actividades del proyecto y horas de ausencia de su unidad productiva. Los piscicultores insistían en que era urgente "pasar de la problemática a la solucionática" (Entrevista abierta. Popayán, Cauca, 20/01/2008). Es decir, los piscicultores esperaban que el proyecto aportara soluciones concretas para los problemas que enfrentaban.

Según los entrevistados, dicha discrepancia fue superada por que los pisciculto- res adoptaron las "nuevas prácticas" que el proyecto defendía (Entrevista abierta. Silvia, Cauca, 10/12/2007). La elaboración participativa de un diagnóstico constituyó uno de los objetivos comunes entre los agentes. Mediante 20 reuniones, realizadas en diferentes localidades del Cauca, de las cuales participaron 130 agentes - piscicultores de pequeña escala, proveedores de insumos, comercializadores y entidades públicas y privadas - se consiguió información primaria actualizada sobre la piscicultura del Cauca. A partir de ese diagnóstico se constató la predominancia del enfoque de subsistencia en la piscicultura del Cauca.

También se logró trazar el perfil de los agentes y la identificación de las relaciones pre-existentes entre ellos. Se identificaron siete organizaciones de productores: Asociación de Productores Piscícolas de Popayán (Asoprop), Asociación de Piscicultores de Silvia (Apropesca), Asociación de Productores Piscícolas de Mindalá (Asopin), Cooperativa de Trabajo de Coconuco (Cootraco), Cooperativa de Piscicultores de Suarez (Tilapaez), Cooperativa Piscícola de Timbio (Coopetim) y la Asociación de Productores Piscícolas del Centro del Cauca (Asopiscec). Esas organizaciones de productores estaban legalmente constituidas, pero prevalecían entre los asociados desconfianzas heredadas de proyectos anteriores. De esa manera, la dinámica productiva, cultural y económica piscícola fue sistematizada a partir de la comprensión de los agentes allí vinculados.

Esos agentes coincidieron al declarar que los siguientes aspectos incidían negativamente en su actividad productiva: a) insipiencia de las organizaciones de productores, constituidas legalmente para facilitar el acceso a proyectos de inversión pública; b) desarticulación con las instituciones locales de fomento; c) carencia del enfoque empresarial y de articulación con el mercadeo; d) desactualización tecnológica de las prácticas de producción, que inciden en la baja productividad y e) limitado acceso a fuentes de crédito y financiación.

Esa identificación colectiva de la situación piscícola del Cauca generó un sentido de responsabilidad y solidaridad reflejadas en la agenda de trabajo y cartera de proyectos que, posteriormente, buscaron articular con la ofer- 
ta de servicios institucionales disponibles en la región. La expresión "ninguno de nosotros es tan fuerte como todos juntos" (Entrevista abierta. Silvia, Cauca, 02/02/2008), expresa pertenencia y entendimiento de que el trabajo conjunto les permitiría cierta representatividad para acceder a los recursos públicos necesarios para el fortalecimiento de la piscicultura del Cauca.

El respeto a los compromisos pactados entre los agentes se destacó en los estudios amparados en el enfoque cultural del capital social. De modo similar, el enfoque estructural también defiende las relaciones sociales de largo plazo como una manifestación de la existencia de capital social. Sin embargo, la línea de base para estimular el trabajo conjunto entre los piscicultores presentaba saldos negativos que se reflejaban en desconfianzas antiguas heredadas en disputas por recursos públicos de fomento a la piscicultura.

Por eso resulta razonable cierto grado de sospecha e incluso de desconfianza entre los agentes en el proceso adelantado. En ese sentido, Foronda y Galindo (2012) alertaron que es erróneo entender la desconfianza como un aspecto negativo cuando se analiza la existencia de capital social. En el caso aquí estudiado, se buscó abordar el recelo existente entre los agentes como un "estado temporal de balance", asumiendo la existencia de las tensiones como inherentes al proceso de consolidación de capital social entre los agentes.

Cimentar capital social, entendido como relaciones duraderas caracterizadas por el respeto a valores como pertenencia y adscripción, no es neural en el horizonte conceptual de cadena productiva citado en este trabajo. Entre tanto, el caso estudiado mostró que esa dinámica constituye una etapa necesaria para que sectores con carácter de subsistencia logren apropiarse del enfoque de cadena productiva. Ante tal constatación, el proyecto aquí analizado requería ser redimensionado temporalmente y respecto a las metas económicas que el enfoque de cadena productiva presupone. No obstante, las entidades financiadoras en Colombia generalmente adoptan el corto plazo - máximo dos años - como plazo para esos proyectos y exigen mejoramiento del desempeño técnico, económico individual y colectivo.
Al finalizar la primera etapa del proyecto, en el año 2006, continuaban siendo incipiente las relaciones comerciales entre los agentes de los distintos eslabones del encadenamiento productivo. Los negocios entre los productores y los proveedores de insumos se limitaban a la compra individual de insumos que, generalmente, era intermediada por agentes (casas agropecuarias) no vinculados a esa iniciativa. La comercialización también continuaba siendo realizada individualmente, predominando la venta al por menor en los mercados locales.

Los agentes entendían que realizando compras individuales no tenían capacidad para incidir en los costos de los insumos. También sabían que su producto final era comercializado a un precio equivalente a los costos de producción, con escasa rentabilidad. Tanto en la compra de insumos como en la comercialización de los productos piscícolas inexistía una estrategia asociativa orientada a incidir en la rentabilidad piscícola. A pesar de la comprensión clara del problema no se logró avanzar en respuestas sostenibles a largo plazo. Por ejemplo, Apropesca, una de las siete asociaciones de productores participante del proceso, avanzó significativamente en la tecnificación de la producción, industrialización y comercialización de trucha. Sin embargo, tales innovaciones no representaron, en la primera etapa del proyecto, incremento en la rentabilidad de dicha asociación de productores.

Otro aspecto identificado en el diagnóstico indicó la desarticulación entre las instituciones locales de fomento y entre ellas y los agentes de la piscicultura del Cauca. La débil presencia de instituciones de apoyo incidía negativamente en los servicios de apoyo a la piscicultura. En ese contexto, la intervención de una institución interfaz fue consolidándose en torno de actividad piscícola. Para Sánchez (1999), la interfaz promueve servicios de apoyo orientados a viabilizar la articulación de los productores al mercado. Es una institución que también actúa como puente entre los técnicos y el tejido empresarial facilitando la adopción de prácticas empresariales.

La capacidad institucional del CREPIC para estimular la articulación estratégica entre los agentes de la piscicultura del Cauca resultó de su creciente interacción con diferentes sectores productivos en Colombia. Desde su 
creación, en el año 2000, había estimulado la apropiación de la gestión de conocimiento en encadenamientos productivos con pequeños productores rurales. El proyecto de fortalecimiento de la piscicultura del Cauca iniciado por el CREPIC en el año 2003, en alguna medida, retomó aprendizajes apropiados por esa institución.

Sin embargo la intervención de la institución interfaz en la pretendida transición en la piscicultura del Cauca no estuvo libre de tensiones. De un lado, fue importante porque soportó institucionalmente el proceso, facilitando el acceso a recursos y potenció el capital relacional entre los agentes del encadenamiento productivo. De otro lado, se evidenció el riesgo de actualizar, en torno de la interfaz, relaciones de dependencia y asistencialismo generalmente atribuidas a los agentes estatales. Es decir, las relaciones de dependencia y cierta subordinación de los agentes de la piscicultura ante las capacidades institucionales y tecnológicas de la institución interfaz, de cierto modo, colocan en evidencia los trazos de una actividad productiva de subsistencia.

La predominancia del carácter de subsistencia exige contextualizar las particularidades del Cauca y de las políticas públicas que han incidido en ese territorio. El Cauca presenta un alto índice de concentración de la tierra, entre 0.73 a 0.98 en el índice de Gini. La mayoría de los municipios presentan un bajo desempeño del índice de necesidades básicas insatisfechas, porcentaje que oscila entre 20 a $80 \%$. La tasa de desempleo alcanza un $11,3 \%$. Esas variables denotan la fragilidad socioeconómica de ese territorio. Sin embargo, la política pública de incentivo a las cadenas productivas relativizó esas especificidades de orden estructural.

No obstante, hay que destacar el dinamismo alcanzado a partir de la articulación interinstitucional en torno de la piscicultura del Cauca. Al vincular nuevos agentes como grupos de investigación, instituciones interfaz y fuentes de financiación se potenciaron resultados e indicadores de desempeño que no son valorados desde la óptica económica tradicional. La articulación interinstitucional surgió en respuesta a las demandas identificadas por los agentes piscícolas. De ese modo, se logró iniciar estrategias de socialización de conocimiento que facilitó el delineamiento de redes de relaciones, más o menos institucionalizadas, entre los piscicultores y sus respectivas asociaciones y entre estas y las instituciones de apoyo a la piscicultura.

\section{Consideraciones finales}

En este trabajo se buscó entender cómo se dinamizó la adopción del enfoque de cadena productiva entre los agentes de la piscicultura del Cauca. En esa dinámica la figura del acuerdo de competitividad se tornó un aspecto diferenciador en la disputa por recursos públicos para financiar el mejoramiento de las actividades productivas rurales. Dicho acuerdo, conforme la Ley $\mathrm{n}^{\circ} 811$ de 2003, constituye un instrumento formal que ratifica la organización de un sector productivo bajo el enfoque de cadena productiva. Es decir, dicho enfoque conquistó su espacio en la medida en que se constituyó en una estrategia diferenciada de acceso a recursos públicos.

La etiqueta "cadena productiva" fue adoptada por los agentes de la piscicultura, a partir de mayo del 2003, al inicio del proyecto aquí estudiado. Esa etiqueta representa un encuadramiento problemático para esos agentes. Si de un lado, esa etiqueta facilitó la participación en procesos de evaluación de proyectos que demandan recursos públicos al ser comparados con cadenas productivas propiamente dichas se encuentra que no poseen indicadores de desempeño exigidos mejoramiento de la distribución del ingreso, aumento de los niveles de competitividad de los agentes y eslabones de la cadena, agregación de valor, entre otros. Esos indicadores enfatizan aspectos económicos que indican que la piscicultura del Cauca continúa siendo una actividad productiva en la cual predomina el enfoque de subsistencia.

El análisis aquí desarrollado mostró que los avances más relevantes, en el proceso que buscó la transición del enfoque de subsistencia para el enfoque de cadena productiva en la piscicultura del Cauca, indican esfuerzos de los agentes que contribuyeron con el fortalecimiento de las redes de relaciones entre ellos y su sentido de pertenencia con las organizaciones existentes en torno de la piscicultura caucana. Es decir, ese proceso estimuló el capital social mediante espacios de reconocimiento y encuentro entre esos agentes. 
Las relaciones entre los agentes de la piscicultura del Cauca se fortalecieron mediante la materialización de acciones conjuntas orientadas a ganar representatividad y viabilizar respuestas para los problemas identificados en el diagnóstico, principalmente, el acceso a recursos tecnológicos y financieros. Para tal propósito fue elaborada una cartera conjunta de proyectos cuya gestión garantizó la continuidad de esa intervención. En esa dinámica se destaca que las redes estables de relaciones individuales e institucionales facilitó el acceso a diferentes recursos potenciando el capital social.

A partir del análisis del caso de la piscicultura del Cauca se verifica que las políticas públicas para sectores productivos con carácter de subsistencia o integrados por pequeños productores pueden ganar densidad explorando las dimensiones del capital social en contextos rurales. En esa lógica es necesario, reconocer las múltiples variables que inciden en la vulnerabilidad de los territorios con agentes que producen bajo el enfoque de subsistencia. De otro lado, ante la inconsistencia de la institucionalidad pública en el ámbito rural las instituciones interfaz muestran su importancia porque además de acompañar y documentar esas dinámicas producen conocimiento sobre las especificidades de cada caso - enfoque de subsistencia o enfoque de cadena productiva.

\section{Referencias}

BOURDIEU, Pierre. Distinctions: a social critique of the judgment of taste. Londrés: Routledge. 1984.

BOLSEGUÍ, M; FUGUET A. Construcción de un modelo conceptual a través de la investigación cualitativa. Revista Sapiens, 7(1), p. 207-229, 2006.

COLOMBIA. Anuario 2003: Observatorio Agrocadenas. Bogotá: Panamericana, 2003. 153p.
COLOMBIA. Convenio de competitividad cadena piscícola nacional. Bogotá: Ministerio de Agricultura y Desarrollo Rural, 2004a. 92p.

Plan estratégico exportador regional cauca. Popayán: CARCE, 2004b. 70p.

- Convenio de competitividad cadena piscícola nacional. Bogotá: Ministerio de Agricultura y Desarrollo Rural, 2004c. 102p.

COMERFORD, John. Reuniões camponesas, sociabilidade e lutas simbólicas. En: PEIRANO, Mariza (Org.). $O$ dito e o feito: ensaios de antropologia dos rituais. Rio de Janeiro: Relume Dumará, 2002.

ESPINAL, F. La cadena de la piscicultura en Colombia. Una mirada global de su estructura y dinámica 1991-2005. Documento de Trabajo $n^{\circ} 72$. Bogotá: Ministerio de Agricultura y Desarrollo Rural, 2005.

FALS BORDA, O. La ciencia y el pueblo: nuevas reflexiones. En: SALAZAR, María Cristina (Ed.). La investigación-acción participativa. Inicios y desarrollo. España: Popular, 1992.

FORONDA, C.; GALINDO, L. Argumentación relativa a la confianza territorial. Claves sobre capital social. Revista Cuadernos de desarrollo rural, 9(68), p. 41-63, 2012.

GAMARRA, José. La economía del departamento del Cauca: concentración de tierras y pobreza. Revista Documentos de trabajo sobre economía regional, (95), 2007.

MORVAN, Y. Filière de Production: Fondaments d'economie industrielle. Paris: Económica, 1985.

ORTIZ, M.; BORJAS, B. La Investigación Acción Participativa: aporte de Fals Borda a la educación popular. Revista Espacio Abierto, 17(4), p. 615-627, 2008.

PIÑONES, S.; ACOSTA, L.; TARTANAC, F. Alianzas Productivas en Agrocadenas. Experiencias de la FAO en América Latina. Santiago de Chile: FAO, 2006.

PUTNAM, R. Making Democracy work. Civic traditions in Modern Italy. New Jersey: Princeton, 1993.

Solo en la bolera. Colapso y resurgimiento de la comunidad norteamericana. Barcelona: Galaxia Gutenberg, 2002.

SÁNCHEZ, P. Política tecnológica para sectores tradicionales: lecciones de los Estados Unidos. Revista Papeles de Economía, 81, p. 242-259, 1999.

TOMTA, D.; CHIATCHOUA, C. Cadenas productivas y productividad de las Mipymes. Revista Criterio Libre, 7(11), p. 145-164, 2009. 